18,13

\title{
Поверхностные плазмон-поляритоны на границе мелкослоистой среды на основе графена
}

\author{
(С) Д.А. Евсеев, Д.И. Семенцов \\ Ульяновский государственный университет, \\ Ульяновск, Россия \\ ฯ E-mail: sementsovdi@mail.ru \\ (Поступила в Редакцию 6 марта 2017 г. \\ В окончательной редакции 20 июня 2017 г.)
}

\begin{abstract}
Исследуются свойства поверхностных плазмон-поляритонов ТМ-типа на границе раздела изотропного диэлектрика и периодической структуры графен-диэлектрик. Установлено, что наличие графена в такой структуре позволяет в определенных частотных диапазонах добиться отрицательности эффективной диэлектрической проницаемости и реализовать условия существования поверхностной волны при практическом отсутствии ее поглощения. Показано влияние доли графена в структуре на характеристики плазмонполяритонов, в частности, на возможность существенного их замедления.
\end{abstract}

Работа выполнена в рамках госзадания Министерства образования и науки РФ (№ 3.6825/БЧ от 2017 г.).

DOI: 10.21883/FTT.2018.03.45569.064

\section{1. Введение}

В последние годы активно исследуются особенности взаимодействия с электромагнитным полем слоев графена и слоистых структур на его основе. Уникальные электронные и оптические свойства (высокие подвижность носителей заряда и проводимость) при практическом отсутствии в широкой частотной области поглощения делают графен одним из наиболее перспективных материалов фотоники и оптоэлектроники. Ввиду особенностей дисперсии проводимости графена физические свойства фотонно-кристаллических структур на его основе могут существенно отличаться от свойств фотонных кристаллов на основе других материалов [1-12]. Существенным также является возможность управления проводимостью графена температурой, электрическим и магнитным полями [13-16].

Для практических применений важную роль играют направляющие свойства графеновых структур. В работах $[17,18]$ была показана возможность распространения локализованных на монослое графена волн, дисперсия которых лежит в терагерцовой частотной области. С целью создания устройств волноводного типа на основе графеновых структур активно исследуется способность двух и более слоев графена, разделенных слоями диэлектрика, удерживать локализованные плазмонные моды [19-24]. В настоящей работе исследуются условия распространения поверхностных плазмон-поляритонов (ПП) ТМ-типа вдоль плоской границы раздела изотропного диэлектрика и периодической структуры, представляющей собой мелкослоистую среду „графен-диэлектрик“. В отличие от [24], где слои графена определяются только мнимой частью проводимости и, следовательно, не учитывается поглощение поверхностных мод, в настоящей работе при анализе используется комплексная проводимость графеновых слоев, в результате чего диэлектрическая проницаемость (ДП) мелкослоистой среды в рассматриваемой области частот является комплексной, а среда - поглощающей.

\section{2. Материальные параметры}

Рассмотрим границу раздела прозрачной подложки из кварцевого стекла с ДП $\varepsilon_{d}=2.15$ и периодической мелкослоистой структуры, которая состоит из чередующихся слоев диэлектрика с ДП $\varepsilon_{1}=5.07$ (оксид кремния) и монослоев графена. Будем считать, что подложка и слои диэлектрика в исследуемом диапазоне частот не обладают дисперсией и что все указанные среды не являются магнитоактивными, поэтому их магнитные проницаемости равны единице. В отличие от [25], где слои графена определяются только мнимой частью проводимости и, следовательно, не учитывается поглощение поверхностных мод, в настоящей работе при анализе используется комплексная проводимость графеновых слоев, в результате чего в рассматриваемой области частот среда в общем случае является поглощающей.

Частотная зависимость комплексной поверхностной проводимости слоев графена $\sigma=\sigma^{\prime}+i \sigma^{\prime \prime}$ определяется выражениями [1-7]

$$
\begin{gathered}
\sigma^{\prime}(\omega)=\sigma_{0} \Theta(\hbar \omega-2 \mu) \\
\sigma^{\prime \prime}(\omega)=\sigma_{0}\left[\frac { 1 } { 2 \pi } \left(\frac{16 k_{\mathrm{B}} T}{\hbar \omega} \ln \left(2 \operatorname{ch}\left(\frac{\mu}{2 k_{\mathrm{B}} T}\right)\right)\right.\right. \\
\left.\left.-\ln \left(\frac{(\hbar \omega+2 \mu)^{2}}{(\hbar \omega-2 \mu)^{2}+\left(2 k_{\mathrm{B}} T\right)^{2}}\right)\right)\right] .
\end{gathered}
$$

Здесь $\sigma_{0}=e^{2} / 4 \hbar \approx 5.5 \cdot 10^{7} \mathrm{~cm} / \mathrm{s}, e-$ заряд электрона, $\hbar$ - постоянная Планка, $\omega$ - частота, $\Theta$ - ступенчатая функция Хэвисайда, $k_{\mathrm{B}}-$ постоянная Больцмана, $T-$ температура, $\mu-$ химический потенциал, $\mu=\hbar v_{\mathrm{F}} \sqrt{\pi n_{0}}$, 


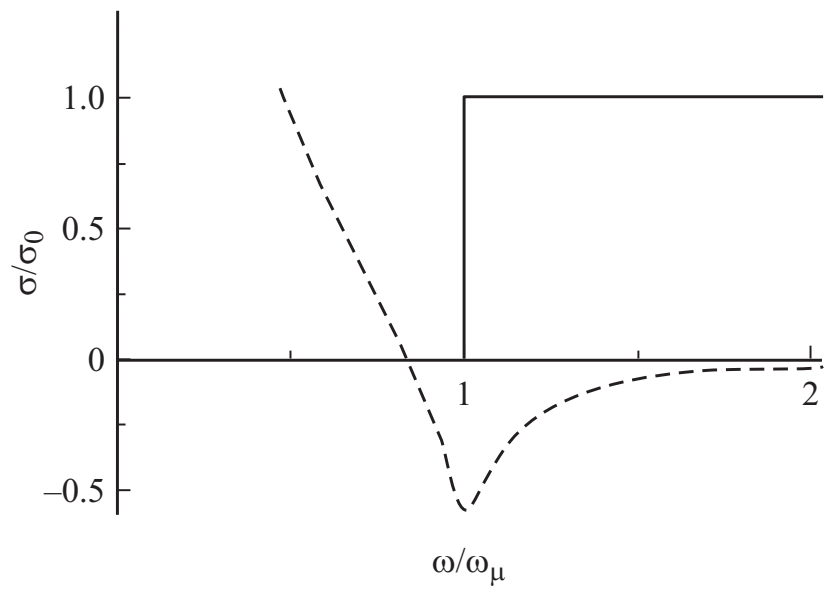

Рис. 1. Частотная зависимость действительной и мнимой части (сплошная и штриховая линии) проводимости графена.

где $n_{0}$ и $v_{\mathrm{F}}$ - концентрация носителей заряда и скорость Ферми в графене.

На рис. 1 представлены частотные зависимости действительной и мнимой частей (сплошные и пунктирные кривые) проводимости графена. Далее при численном анализе используются следующие значения параметров графена: $v_{\mathrm{F}}=10^{8} \mathrm{~cm} / \mathrm{s}, n_{0}=10^{11} \mathrm{~cm}^{-2}, \mu=0.6 \mathrm{eV}$, $\omega_{\mu}=2 \mu / \hbar=1.83 \cdot 10^{15} \mathrm{~s}^{-1}, T=300 \mathrm{~K}$ [5-8]. Видно, что действительная часть проводимости $\sigma^{\prime}$ при $\omega=\omega_{\mu}$ испытывает скачек от нулевого значения до значения $\sigma_{0}$. В области скачка величины $\sigma^{\prime}$ мнимая часть проводимости $\sigma^{\prime \prime}$ достигает минимума. При этом $\sigma^{\prime \prime}$ в достаточно широкой частотной области принимает отрицательные значения, что указывает на возможность реализации в планарной периодической структуре на основе графеновых монослоев отрицательных значений действительной части эффективной ДП.

Рассматриваемая структура диэлектрик-графен обладает выделенным направлением, которым является ось периодичности (ось $0 Z$ ). Наличие выделенного направления указывает на одноосную анизотропию структуры.

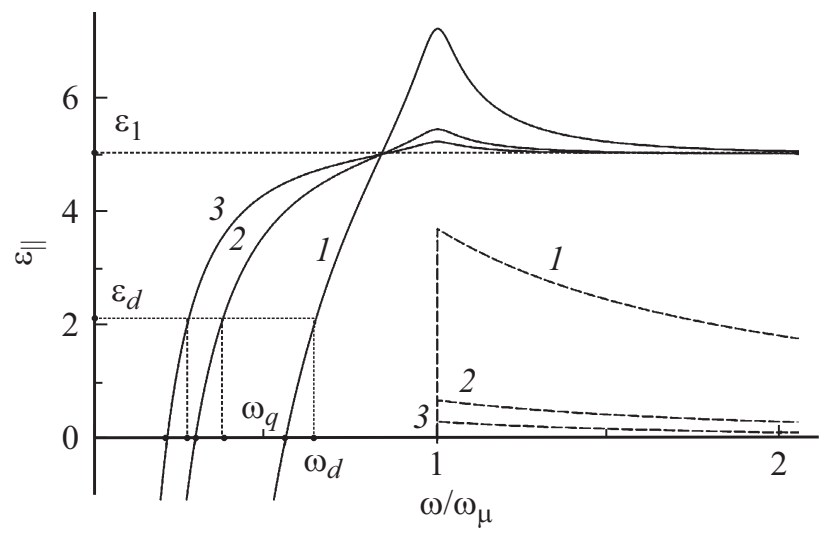

Рис. 2. Частотная зависимость действительной и мнимой части (сплошная и штриховая линии) ДП слоистой структуры диэлектрик-графен с периодом $d=1,510 \mathrm{~nm}$ (кривые 1,2,3).
Компоненты диагонального тензора эффективной ДП такой структуры $\varepsilon_{x}=\varepsilon_{y}=\varepsilon_{\|}, \varepsilon_{z}=\varepsilon_{\perp}$ получены в работах [19-21] и имеют вид

$$
\begin{gathered}
\varepsilon_{\|}=\varepsilon_{\|}^{\prime}+i \varepsilon_{\|}^{\prime \prime}=\varepsilon_{1}+i \frac{4 \pi \sigma}{\omega d}, \quad \varepsilon_{\perp}=\varepsilon_{1}, \\
\varepsilon_{\|}^{\prime}=\varepsilon_{1}-\frac{4 \pi \sigma^{\prime \prime}}{\omega d}, \quad \varepsilon_{\|}^{\prime \prime}=\frac{4 \pi \sigma^{\prime}}{\omega_{d}} .
\end{gathered}
$$

На рис. 2 представлены частотные зависимости действительной и мнимой частей (сплошная и штриховая линии) компоненты $\varepsilon_{\|}$для структуры с периодом $d=d_{1}+d_{2}=(1,5,10) \mathrm{nm}$ (кривые $\left.1-3\right)$, где толщина монослоя графена $d_{2}=0.335 \mathrm{~nm}$. Видно, что на частоте $\omega_{q}$ величина $\varepsilon_{\|}^{\prime}$ обращается в ноль. При этом $\varepsilon_{\|}^{\prime}<0$ в области $\omega<\omega_{q}$, наличие которой указывает на возможность существования в структуре поверхностной волны. Характерными частотами, определяющими поведение волны в структуре, также являются: частота $\omega_{d}$, которая является решением уравнения $\varepsilon_{\|}^{\prime}=\varepsilon_{d}$, и частота $\omega_{s}$, которая существенно влияет на характер дисперсии распространяющихся волн и является решением уравнения $\varepsilon_{\|}^{\prime}=\varepsilon_{d}^{2} / \varepsilon_{\perp}$. На рисунке приведена частота $\omega_{d}$ (для одного из значений периода $d$ ). Уменьшение периода структуры (т.е. увеличение доли графена) приводит к увеличению указанных частот. В области скачка величины $\sigma^{\prime}$ величина $\varepsilon_{\|}^{\prime}$ достигает максимального значения. C уменьшением периода структуры растут максимальные значения как реальной, так и мнимой части эффективной ДП. В дальнейшем мы будем интересоваться только областью $\omega<\omega_{\mu}$, где в слоистой структуре отсутствует поглощение, так как величина мнимой части ее ДП $\varepsilon_{\|}^{\prime \prime}=0$. Наличие такой области существенно отличает рассматриваемую проводящую структуру на основе графена от структуры металл-диэлектрик.

\section{3. Дисперсионное соотношение}

Будем считать, что поверхностный ПП распространяется вдоль оси $0 X$. В данной структуре поверхностными могут быть ПП только ТМ-типа с компонентами поля $\left(E_{x}, H_{y}, E_{z}\right)$. При этом все компоненты волнового поля зависят от времени и продольной координаты пропорционально фактору $\exp [i(\omega t-\beta x)]$, где $\beta-$ константа распространения. Зависимость компонент волнового поля от поперечной координаты определяется уравнениями

$$
\begin{gathered}
\frac{d^{2} H_{y}}{d z^{2}}-q_{j}^{2} H_{y}=0, \quad E_{z}=-\frac{\beta}{k_{0} \varepsilon_{z j}} H_{y}, \\
E_{x}=\frac{1}{i k_{0} \varepsilon_{x j}} \frac{d}{d z} H_{y},
\end{gathered}
$$

где $j=d, s$, поперечные компоненты волнового вектора в каждой из сред

$$
q_{d}^{2}=\beta^{2}-k_{0}^{2} \varepsilon_{d}, \quad q_{s}^{2}=\varepsilon_{\|}\left(\beta^{2} / \varepsilon_{\perp}-k_{0}^{2}\right),
$$

$k_{0}=\omega / c, c-$ скорость света в вакууме. В общем случае и константа распространения, и поперечные компоненты волнового вектора являются комплексными величинами, т.е. $\beta=\beta^{\prime}+i \beta^{\prime \prime}$ и $q_{j}=q_{j}^{\prime}+i q_{j}^{\prime \prime}$. Решение 
первого из уравнений (4) с учетом непрерывности компоненты $H_{y}$ на границе раздела сред приводит к следующей зависимости от координаты $z$ магнитного поля ПП

$$
H_{y}(z)=H_{0} \begin{cases}\exp \left(q_{s}^{\prime} z\right) \exp \left(i q_{s}^{\prime \prime} z\right), & z<0 \\ \exp \left(-q_{d}^{\prime} z\right) \exp \left(-i q_{d}^{\prime \prime} z\right), & z>0\end{cases}
$$

где $H_{0}-$ амплитуда магнитного поля при $z=0$. Компоненты электрического поля находятся с помощью подстановки соотношения (5) в уравнения (3).

Необходимым условием существования поверхностного ПП, т.е. локализованного режима распространения, является выполнение в каждой из сред неравенств $q_{d, s}^{\prime}>0$, которые обеспечивают экспоненциальный спад амплитуды волнового поля при удалении от границы раздела. Также требуется выполнение неравенств $\beta^{\prime}>0$ и $\beta^{\prime \prime}>0$, первое из которых указывает на положительность фазовой скорости ПП, второе - на отсутствие его усиления.

Так как при $\omega<\omega_{\mu}$ величина $\varepsilon_{\|}^{\prime \prime}=0$, то в области существования поверхностного ПП мнимая часть константы распространения $\beta^{\prime \prime}=0$, а действительная часть определяется следующим дисперсионным соотношением

$$
\beta^{\prime}=k_{0} \sqrt{\varepsilon_{d} \varepsilon_{\perp} \frac{\varepsilon_{d}-\varepsilon_{\|}^{\prime}}{\varepsilon_{d}^{2}-\varepsilon_{\perp} \varepsilon_{\|}^{\prime}}}
$$

Видно, что при $\varepsilon_{\|}^{\prime}<\varepsilon_{d}^{2} / \varepsilon_{s}$ и $\varepsilon_{\|}^{\prime}>\varepsilon_{d}$, что соответствует областям $\omega<\omega_{s}$ и $\omega>\omega_{d}$, подкоренное выражение в (6) является положительной величиной и $\beta^{\prime}$ принимает действительные значения. В области от $\omega_{s}$ до $\omega_{d}$ подкоренное выражение принимает отрицательные значения и эта область является запрещенной, т. е. в этой области $\beta^{\prime \prime} \neq 0$. Как будет показано далее (при анализе поперечных компонент волнового вектора) распространяющийся в структуре ПП является локализованным лишь в области частот $\omega<\omega_{s}$.

На рис. 3 приведены частотные зависимости действительной и мнимой части константы распространения, нормированной на величину $k_{\mu}=\omega_{\mu} / c=6.1 \cdot 10^{4} \mathrm{~cm}^{-1}$. Указанные величины получены для значений периода структуры $d=(1,5,10) \mathrm{nm}$ (кривые $1,2,3)$, которые будут использоваться далее. В области частот $\omega<\omega_{s}$ дисперсионная кривая определяет поведение поверхностного ПП. Так как в этой области поглощение отсутствует, то длина пробега ПП $L=1 / \beta^{\prime \prime}$ в структуре оказывается неограниченной. С приближением частоты к $\omega_{s}$ наблюдается асимптотический рост величины $\beta^{\prime}$, что указывает на резкое уменьшение длины волны $\lambda=2 \pi / \beta^{\prime}$ распространяющегося ПП. С увеличением периода структуры частота $\omega_{s}$ смещается в область более низких частот. Область частот $\omega_{s}<\omega<\omega_{d}$ является запрещенной, так как в ней отлична от нуля лишь мнимая часть константы распространения, которая определяет длину затухания пробега ПП $L$. В области $\omega>\omega_{d}$ в структуре может распространяться только объемный ПП, поведение которого определяется дисперсионной кривой в этой области.

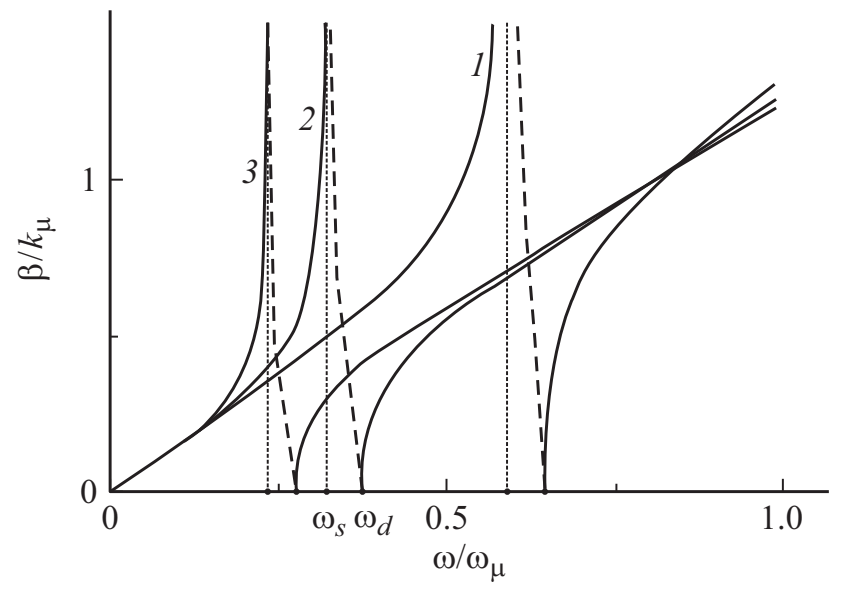

Рис. 3. Частотная зависимость реальной и мнимой части (сплошная и штриховая линии) константы распространения, $d=1,5,10 \mathrm{~nm}$ (кривые $1,2,3)$
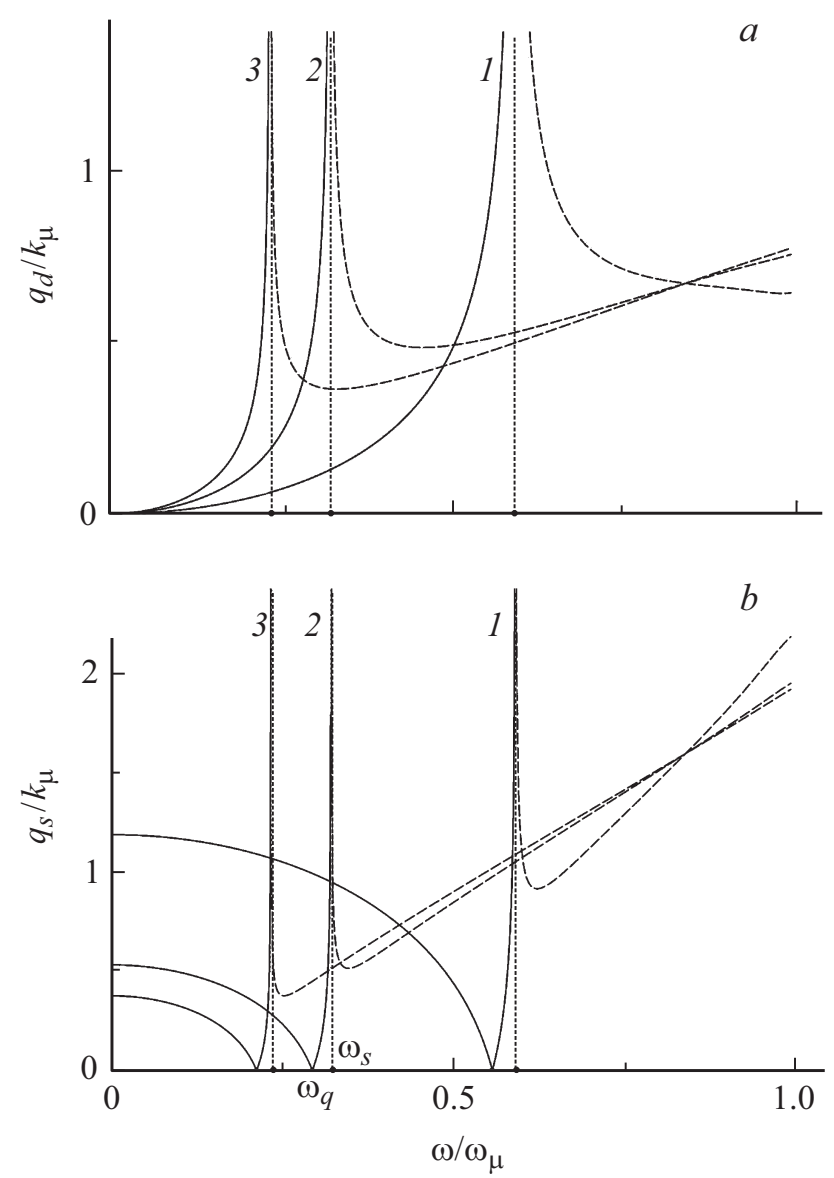

Рис. 4. Частотная зависимость действительной и мнимой части (сплошная и штриховая линии) поперечной компоненты волнового вектора в диэлектрике $(a)$ и слоистой структуре $(b)$, $d=1,5,10 \mathrm{~nm}$ (кривые 1,2,3).

На рис. 4 приведены частотные зависимости действительной и мнимой части (сплошные и штриховые линии) поперечной компоненты волнового вектора в диэлектрике и слоистой среде. В обеих средах ПП 


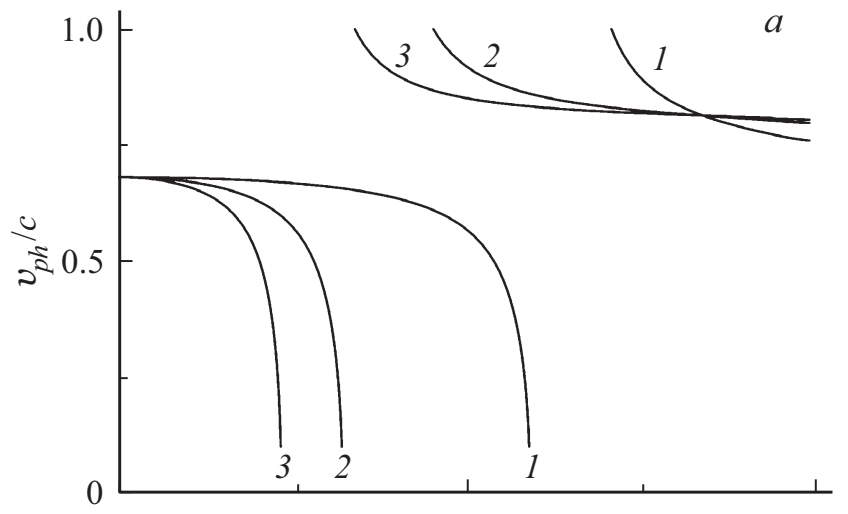

$b$

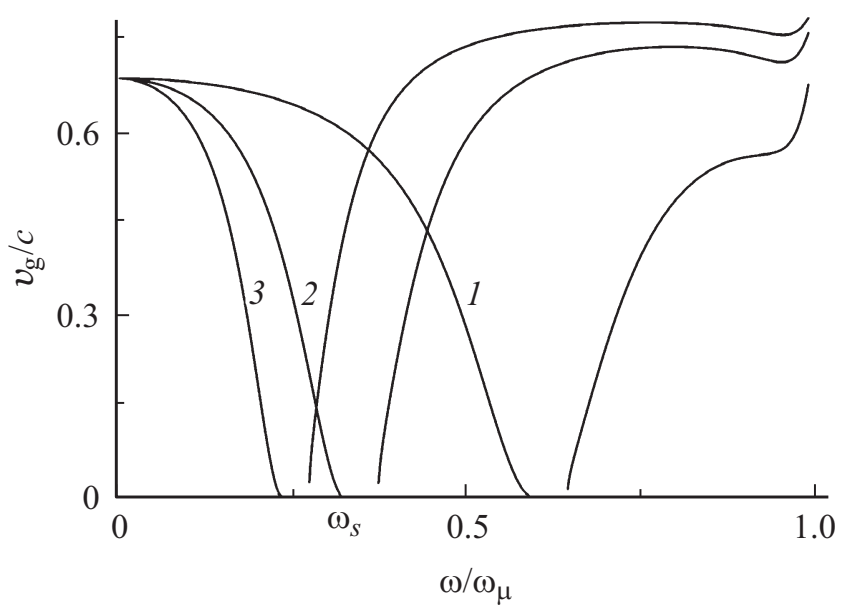

Рис. 5. Частотная зависимость фазовой $(a)$ и групповой скорости ПП $(b)$.

является локализованным вблизи границы в области $\omega<\omega_{s}$. Глубина проникновения ПП в диэлектрик и слоистую среду определяется величиной $\delta_{d}=1 / q_{d}^{\prime}$ и $\delta_{s}=1 / q_{s}^{\prime}$. При $\omega \rightarrow \omega_{q}$ величина $q_{s}^{\prime} \rightarrow 0$, что указывает на отсутствие локализации ПП в слоистой среде на указанной частоте. При дальнейшем увеличении частоты ПП вновь становится локализованным в обеих средах и при $\omega \rightarrow \omega_{s}$ величины $\delta_{s}$ и $\delta_{d}$ стремятся к нулю, что указывает на максимальную локализацию ПП на границе раздела сред вблизи указанной частоты. При $\omega>\omega_{s}$ ПП становится объемным в каждой из сред, так как в этой области $q_{d, s}^{\prime}=0$, а $q_{d, s}^{\prime \prime} \neq 0$.

На рис. 5 представлены частотные зависимости групповой скорости $v_{g}=d \omega / d \beta^{\prime}$ и фазовой скорости $v_{\mathrm{ph}}=\omega / \beta^{\prime}$ ПП, распространяющегося вдоль границы раздела сред. Видно, что для локализованного ПП с ростом частоты групповая скорость падает и с приближением частоты к $\omega_{s}$ ПП испытывает существенное замедление. Область частот $\omega_{s}<\omega<\omega_{d}$ является запретной, поэтому в структуре в этой области нет распространяющихся волн. В области $\omega>\omega_{d}$ в структуре может распространяться только объемный ПП, поведение которого определяется дисперсионной кривой в этой области. С уменьшением доли графена в структуре частотный интервал, на котором происходит убывание скорости, уменьшается. Фазовая скорость ПП также уменьшается при приближении частоты к $\omega_{s}$, и чем больше доля графена в структуре, тем быстрее происходит это уменьшение.

\section{4. Энергетические потоки}

Энергетической характеристикой волнового процесса с учетом его гармонической зависимости от времени является вектор Пойнтинга $\langle\mathbf{S}\rangle=(c / 8 \pi) \cdot \operatorname{Re}\left(\mathbf{E} \times \mathbf{H}^{*}\right)[24]$, определяющий в рассматриваемом нами случае среднюю за период плотность потока энергии ПВ. Наличие как поперечной, так и продольной волновой компонент электрического поля приводит к тому, что вектор $\langle\mathbf{S}\rangle$ имеет как продольную $\left\langle S_{x}\right\rangle$, так и поперечную $\left\langle S_{z}\right\rangle$ составляющие. С учетом соотношений для полей (3) и (5), запишем выражения для продольной компоненты $\left\langle S_{x}(x, z)\right\rangle=S_{x}(z) \exp \left(-2 \beta^{\prime \prime} x\right)$, которая определяет перенос энергии волны вдоль направления распространения в каждой из сред

$$
S_{x}(z)=S_{0} \begin{cases}\left(\beta^{\prime} / k_{0} \varepsilon_{d}\right) \exp \left(-2 q_{d}^{\prime} z\right), & z>0, \\ \left(\beta^{\prime} / k_{0} \varepsilon_{\perp}\right) \exp \left(2 q_{s}^{\prime} z\right), & z<0 .\end{cases}
$$

На рис. 6 представлены частотные зависимости компоненты $S_{x}(z)$ (нормированной на величину $S_{0}=c H_{0}^{2} / 8 \pi$ ). Сплошным линиям отвечают зависимости плотности потока в диэлектрике вблизи границы раздела сред (при $\left.z>0_{+}\right)$, пунктирным линиям - зависимости в слоистой среде (при $z>0_{-}$). Видно, что в диэлектрике плотность потока выше, чем в слоистой среде. С приближением частоты к $\omega_{x}$ наблюдается асимптотический рост плотности потока ПВ. При $\omega \geq \omega_{d}$ наблюдается рост плотности потока объемной волны (начиная с нулевого значения) с дальнейшим выходом на насыщение.

Важной величиной поверхностной волны является величина продольной компоненты полного потока энергии $P_{x}$, переносимой распространяющейся в структуре волной. Эта величина находится в результате интегрирования компоненты $\left\langle S_{x}\right\rangle$ по всему поперечному сечению структуры

$$
\begin{aligned}
P_{x}(x) & =\int_{-\infty}^{\infty}\left\langle S_{x}(x, z)\right\rangle d z \\
& =P_{0} \frac{k_{\mu}}{k_{0}}\left(\frac{\beta^{\prime}}{\varepsilon_{1} q_{s}^{\prime}}+\frac{\beta^{\prime}}{\varepsilon_{d} q_{d}^{\prime}}\right) \exp \left(-2 \beta^{\prime \prime} x\right) .
\end{aligned}
$$

На рис. 7 представлены частотные зависимости продольной компоненты полного энергетического потока (нормированного на величину $P_{0}=S_{0} / k_{\mu}$ ), полученные для ПВ в области ее существования. Как следует из рисунка, значения продольной компоненты потока энергии максимальны вблизи частоты $\omega_{q}$, а наименьшего значения достигают на частоте $\omega_{s}$, выше которой (до частоты $\omega_{d}$ ) волны в структуре не распространяются. Так как в области частот $\omega<\omega_{s}$ поглощение отсутствует $\left(\beta^{\prime \prime}=0\right)$, то в рассматриваемой структуре ПВ 


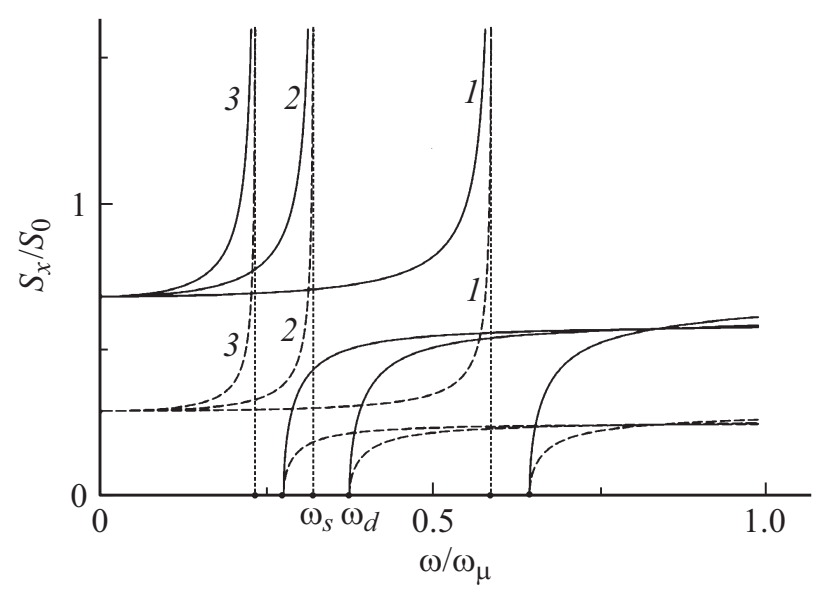

Рис. 6. Частотная зависимость плотности потока энергии в диэлектрике и слоистой среде (сплошная и штриховая линии).

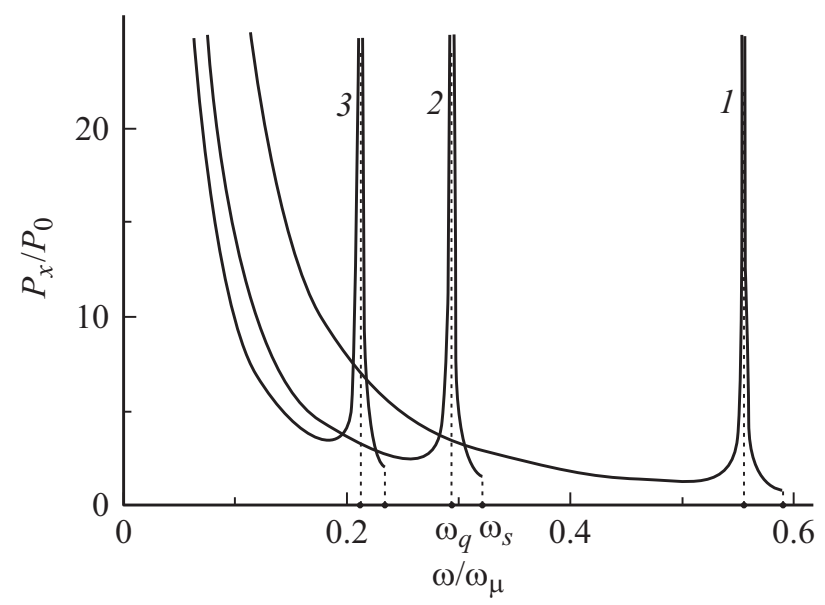

Рис. 7. Частотная зависимость полного потока энергии ПП в структуре.

распространяется без затухания. В конечном счете, это связано с характерной особенностью графена, для которого $\sigma^{\prime}=0$ в области $\omega<\omega_{\mu}$.

\section{5. Заключение}

В работе исследованы особенности распространения поверхностной и объемной волны ТМ поляризации вдоль плоской границы раздела изотопного диэлектрика и мелкослоистой структуры, состоящей из чередующихся слоев графена и диэлектрика. В результате решения уравнений электромагнитного поля совместно с граничными условиями получены выражения для электрического и магнитного волновых полей в структуре, дисперсионное соотношение для собственной ТМ волны, выражения для плотности потока и полного потока энергии, переносимой волной. Для различных значений периода структуры (фактически, толщины диэлектрических слоев между соседними слоями графена) получены частотные зависимости отличных от нуля диагональных компонент тензора эффективной ДП. На основе чис- ленного анализа дисперсионного соотношения построены частотные зависимости константы распространения, групповой и фазовой скоростей, энергетических потоков. Показано наличие двух частотных областей, в одной из которых реализуется режим распространения ПВ, во второй - объемной (излучательный). Показано, что наличие графена в структуре позволяет реализовать условия существования поверхностных волн при практическом отсутствии поглощения. Изучено влияние доли графена в структуре на характеристики поверхностной волны, показана возможность существенного ее замедления. Проведенный анализ указывает на возможность управления дисперсионными характеристиками ПВ за счет изменения материала подложки или диэлектрика в слоистой структуре.

\section{Список литературы}

[1] С.В. Морозов, К.С. Новоселов, А.К. Гейм. УФН 178, 776 (2008).

[2] A.H. Castro Neto, F. Guinea, N.M.R. Peres, K.S. Novoselov, A.K. Geim. Rev. Mod. Phys. 81, 109 (2009).

[3] L.A. Falkovsky, A.A. Varlamov. Eur. Phys. J. B 56, 281, (2007).

[4] L.A. Falkovsky, S.S. Pershoguba. Phys. Rev. B. 76, 153410 (2007).

[5] G.W. Hanson. J. Appl. Phys. 103, 064302 (2008).

[6] V.Ya. Aleshkin, A.A. Dubinov, V. Ryzhii. Pis'ma v ZhETF. 89, 2, 70 (2009).

[7] A.S. El-Naggar. Springer Science, Opt. Quant. Electron. DOI 10. 1007/s11082-014-0021-1 (2014).

[8] M.I. Katsnelson. Eur. Lett. 84, 37001 (2008).

[9] O.L. Berman, R.Ya. Kezerashvili. J. Phys.: Condens. Mater. 24, 015305 (2012).

[10] A. Madani, S.R. Entezar. Phys. B 431, 1 (2013).

[11] А.А. Колесников, Ю.Е. Лозовик. Тр. МФТИ. 5, 1, 53 (2013).

[12] H. Liang, S. Ruan, M. Zhang, H. Su, I. Ling Li. Appl. Phys. Lett. 107, 091602 (2015).

[13] O.L. Berman, G. Gumbs, Yu.E. Lozovik. Phys. Rev. B. 78, 085401 (2008).

[14] A. Ferreira, J. Viana-Gomes, Yu.V. Bludov, V.M. Pereira, N.M.R. Peres, A.H. Castro Neto. arXiv: 1109, 3188 (2012).

[15] A.V. Babichev, V.E. Gasumyants, V.Y. Butko. J. Appl. Phys. 113, 7, 076101 (2013).

[16] А.В. Пак, М.Б. Белоненко. ФТТ 55, 822 (2013).

[17] C.H. Gan, H.S. Chu, E.P. Li. Phys. Rev. B 85, 125431 (2012).

[18] Ю.Е. Лозовик. УФН. 182, 1111 (2012).

[19] B. Zhu, G. Ren, S. Zheng, Z. Lin, S. Jian. Opt. Express. 21, 17089 (2013).

[20] D. Svintsov, V. Vyurkov, V. Ryzhii, T. Otsuji. arXiv: arXiv: 1211, 3629 (2012).

[21] М.Б. Белоненко, Н.Г. Лебедев, Н.Н. Янюшкина. ФТТ 54, 1, 162 (2012).

[22] П.И. Буслаев, И.В. Иорш, И.В. Шадривов, П.А. Белов, Ю.С. Кившарь. Письма в ЖЭТФ 97, 619 (2013).

[23] D. Smirnova, P. Buslaev, I. Iorsh, I.V. Shadrivov, P.A. Belov, Yu.S. Kivshar. Phys. Rev. B. 89, 245414 (2014)

[24] B. Zhu, G. Ren, S. Zheng, Z. Lin, S. Jian. Opt. Express. 21, 17089 (2008).

[25] Л.Д. Ландау, Е.М. Лифшиц. Электродинамика сплошных сред. Наука, М., (1982). 621 с. 